Original Paper $\quad$ http://ajol.info/index.php/ijbcs http://indexmedicus.afro.who.int

\title{
Etude de la dynamique de la macrofaune du sol sous culture de deux variétés de patate douce (Ipomea batatas) avec utilisation de différents modes de désherbage
}

\author{
Mamoudou TRAORE ${ }^{1 *}$, Awa BARRO ${ }^{2}$, Ali GARANE ${ }^{3}$ et NACRO Hassan Bismarck ${ }^{2}$ \\ ${ }^{1 *}$ Institut de l'Environnement et de Recherches Agricoles (INERA), CREAFK, Département Gestion des \\ Ressources Naturelles/Système de Production, 03 BP 7047 Ouaga 03, Burkina Faso. \\ ${ }^{2}$ Universite Polytechnique de Bobo-Dioulasso, Institut du Développement Rural; Laboratoire d'Etude et de \\ Recherche sur la Fertilité du Sol, 01 BP 1091 Bobo-Dioulasso, Burkina Faso. \\ ${ }^{3}$ Institut de l'Environnement et de Recherches Agricoles (INERA), Département Production Végétale, 01 BP \\ 470 Ouagadougou 01, Burkina Faso. \\ "Auteur correspondant ; E-mail : tramadalbela@yahoo.fr
}

\section{REMERCIEMENTS}

Nous remercions le Laboratoire d'Etude et de Recherche sur la Fertilité du Sol (LERF) de l'Institut du Développement Rural de l'Université Polytechnique de Bobo-Dioulasso pour la prise en charge financière de cette étude.

\section{RESUME}

Les communautés de la macrofaune du sol sous culture de deux variétés de patate douce (BF 11 et Ejumula 2) ont été inventoriées huit semaines après le repiquage des boutures. Les expérimentations ont été conduites en 2014 sur des parcelles du Centre de Formation et de Recherches Environnementales et Agricoles et de Formation de Kamboinsé de l'Institut de l'Environnement et de Recherches Agricoles (INERA). L'objectif de cette étude était d'identifier les différentes espèces de la faune du sol et de déterminer l'influence des différentes pratiques agricoles sur la faune du sol. Les termites ont été échantillonnés par la méthode des monolithes et par transect ; les vers de terre ont été échantillonnés uniquement par monolithes. Au total, quinze (15) espèces d'insectes et deux (02) espèces de vers de terre ont été enregistrées sous les traitements de la variété F0. Pour les insectes, les espèces identifiées sont : Microtermes pusillus, Feronia sp, Capsus sp, Amara sp, Amara auliae, Philontus marginatus, Dromius sp, Dromius quadrimaculatus, Xestobium sp, Pachycondyla analis, Paederus sp, Trinervitermes sp, Monyomorium abyssinicumi, Dyschirius globusus, Amitermes stephensoni. Les vers de terre identifiés sont Milsonia inermis et Dichogaster affininis. Les traitements sans désherbage ou désherbage tardif (après la huitième semaine) ont connu une meilleure installation de la macrofaune du sol. Les termites ont été les organismes favorisés dans les cas d'absence totale de désherbage. Le type de variété de patate douce n'a pas eu d'effet sur les communautés des la macrofaune du sol. (C) 2015 International Formulae Group. All rights reserved.

Mots clé : Patate douce, macrofaune du sol, désherbage, traitement. 


\title{
Study of the dynamics of soil macrofauna under cultivation of two varieties of sweet potato (Ipomoea batatas) using different modes of weeding
}

\begin{abstract}
The communities of soil macrofauna under cultivation of two varieties of sweet potato (BF 11 and Ejumula 2) were surveyed eight weeks after cuttings transplanting. The experiments were conducted in 2014 on plots of the Training Centre for Environmental Research and Agricultural of Kamboinsé, Training Institute for the Environment and Agricultural Research (INERA). The objective of this study was to identify the different species of soil fauna and determine the influence of different agricultural practices on soil fauna. The termites were sampled by the monoliths and transect method; earthworms were sampled only by monoliths. Fifteen (15) species of insects and two (02) species of earthworm have been registered in the treatment of F0 variety. For insects, the species identified are: Microtermes pusillus, Feronia sp Capsus sp sp Amara, Amara auliae, Philontus marginatus, Dromius sp Dromius quadrimaculatus Xestobium sp Pachycondyla analis, Paederus sp sp Trinervitermes, Monyomorium abyssinicumi, Dyschirius globosus, Amitermes stephensoni. Earthworms identified are Milsonia inermis and Dichogaster affininis. The treatment without weeding or late weeding (after the eighth week) had a better installation of the soil macrofauna. Termites were the favored organisms in the event of total absence of weeding. The type of variety of sweet potato hadn't any effect on the communities of soil macrofauna.
\end{abstract}

() 2015 International Formulae Group. All rights reserved.

Keywords: Sweet potato, soil macrofauna, weeding, treatment.

\section{INTRODUCTION}

Le grand défi actuel pour le développement de l'agriculture est de conserver la biodiversité et développer l'usage des ressources biologiques complètement négligées pendant des décennies, comme c'est le cas des invertébrés du sol (Lavelle et Fragoso, 2000; Decaëns et al., 2006 ; Zangerlé, 2011). Parmi les actions qui concourent à la protection du sol, un grand nombre d'auteurs attribuent un rôle prépondérant aux invertébrés du sol, qui sont des indicateurs très sensibles de la qualité des sols et de leur fertilité (Lavelle et al., 2006 ; Rombké et al., 2006; Peres et al., 2008; Chaudhuri et al., 2012). Pour ces auteurs, la qualité biologique des sols fait référence à l'abondance, à la diversité et à l'activité des organismes vivants qui participent au fonctionnement du sol. Ainsi, la non durabilité générale actuelle des systèmes agricoles mondiaux provient, au moins en partie, selon nombre d'auteurs, de la disparition des invertébrés du sol (Wolters, 2001; Barros et al. 2004; Huhta, 2006; Blouina et al., 2013 ; Rousseau et al., 2013).
Parmi ces invertébrés, la macrofaune du sol constitue une ressource qui remplit au sein des écosystèmes, des fonctions essentielles pour le maintien de la qualité des sols (Wolters 2001 ; Lavelle et al., 2006 ; Jouquet et al., 2007). Les " organismes ingénieurs ", fourmis, termites et vers de terre vivent principalement dans le sol et créent de nombreuses structures (pores, galeries et agrégats) qui entretiennent les propriétés hydrauliques des sols (infiltration et rétention de l'eau, notamment) (Ruiz Camacho, 2004; Lavelle et al., 2006; Zangerlé, 2011). Pour Jouquet et al. (2014), la macrofaune peut être utilisée pour restaurer les fonctions de l'écosystème. Pour nombre d'auteurs, les vers de terre constituent un rempart contre les nématodes phytoparasites des cultures (Lafont et al., 2007 ; Boyer et al., 2013). Ainsi, la qualité des sols doit faire référence, à l'abondance, à la diversité et à l'activité des organismes vivants qui participent du fonctionnement du sol (Rombké et al., 2006; Rossi, 2006; Jouquet et al., 2011).

L'objectif global de cette étude était d'étudier la dynamique de la faune du sol sous 
culture de la patate douce. La patate douce (Ipomea batatas) est une convolvulacée vivace cultivée comme une plante annuelle. Les tiges rampantes aux extrémités dressées, supportent des feuilles alternes polymorphes, à découpures nettes, d'un vert violacé et des fleurs à style capité et à corolle infundibuliforme de couleur violette ou blanche, groupées en ombelles axillaires. Le fruit est une capsule indéhiscente comportant une ou deux graines semblables à celles du liseron; graines qu'on voit rarement car la plante fructifie peu en culture. Le système radiculaire fasciculé donne naissance à des racines qui s'incurvent vers le bas en formant des tubercules de formes et de couleurs de chair variables (blanches, jaunes, rouges ou violettes) dont le poids peut atteindre $3 \mathrm{~kg}$. Ces tubercules légèrement sucrés car riches en glucides et en fécule, mais pauvres en protides, sont comestibles.

$$
\text { Il s'agissait d'une manière }
$$
spécifique (i) d'identifier les différentes espèces de la faune du sol et de (ii) déterminer l'influence des différentes pratiques agricoles sur la faune du sol.

\section{MATERIEL ET METHODES \\ Le matériel végétal}

Le matériel végétal utilisé était constitué par une variété de patate douce à chair blanche (BF 11) et une autre à chair orange (Ejumula 2). La variété BF 11, d'origine locale, a un cycle court de 100-120 jours. Les entre-nœuds sont longs et la tige poilue de couleur rouge est très vigoureuse, ce qui lui assure une bonne reprise après plantation. Le feuillage de forme ovale est homogène et très envahissant. La peau et la chair des tubercules sont blanches. Cette variété est très sensible aux attaques de charançons (Cylas formicarius). La variété BF 11 se cultive aussi bien en saison chaude qu'en saison fraîche. La productivité est moyenne. Les tubercules ont tendance à éclater en fin de cycle. Pour éviter cela, il convient d'éviter les récoltes trop tardives.
La variété Ejumula 2 a été obtenue à partir des graines collectées sur la variété locale Ougandaise Ejumula (C.I.P 443750). Elle a également un cycle court de 100-120 jours. La tige de couleur blanche est poilue avec des entre-nœuds courts. Le feuillage est un peu hétérogène et peu envahissant. Les tubercules ont une forme homogène avec une peau est de couleur blanche. La chair est de couleur crème à l'extérieur mais orange foncée à l'intérieur, très sucrée et de bonne qualité gustative. La variété est très sensible aux charançons (Cylas formicarius) et aux maladies virales. La productivité de la variété Ejumula 2 est très bonne. Cette variété se cultive également aussi bien en saison sèche qu'en saison fraîche. La reprise des tiges, grosses en générale est très bonne après plantation.

\section{Dispositif expérimental}

Les expérimentations ont été conduites en 2014 sur des parcelles du Centre de Formation et de Recherches Environnementales et Agricoles et de Formation de Kamboinsé (CREAF-K) de l'Institut de l'Environnement et de Recherches Agricoles (INERA). La parcelle élémentaire de $10,5 \mathrm{~m}^{2}$ constituée de 3 billons de $3,5 \mathrm{~m}$ de long chacun avec un espacement de $1 \mathrm{~m}$ entre billons consécutifs soit une parcelle utile de 3,5 $\mathrm{m}^{2}$ constituée par le billon central. Le nombre de répétition est de 4 pour chacune des variétés. Par variété, les parcelles étaient au nombre de 24 (6 traitements x 4), soit un nombre total de 48 parcelles pour l'essai. La superficie utile de l'essai est $168 \mathrm{~m}^{2}$ (48 m x $3,5 \mathrm{~m})$ pour une superficie totale de $636 \mathrm{~m}^{2}$ (12 m x $53 \mathrm{~m})$ ou encore (48 mx 10,5 m +12 $\mathrm{m} \times 11 \mathrm{~m})$. Sur chaque billon, les boutures ont été plantées à un écartement de $30 \mathrm{~cm}$ entre boutures consécutives soit 12 boutures sur le billon et un nombre total de 864 boutures [( 24 x 3 billons) x 12)] pour chacune des variétés. Le nombre total de boutures pour l'essai est de 1728 .

La fertilisation adoptée est celle obtenue après 3 années d'essai à la station du 
CREAF-Kamboinsé constituée par un apport de fumure organique bien décomposée au labour à la dose de $20 \mathrm{t} / \mathrm{ha}\left(20 \mathrm{~kg} / 10 \mathrm{~m}^{2}\right.$ ou 7 $\mathrm{kg} / \mathrm{billon}$ de $3,5 \mathrm{~m}$ ) et un apport d'engrais NPK (14-23-14) est fait à 21 jours après repiquage à la dose de $350 \mathrm{~kg} / \mathrm{ha}(122,5 \mathrm{~g}$ par billon de 3,5 m).

Pour la protection phytosanitaire contre les charançons au moment du repiquage dans la nouvelle parcelle, les boutures ont été trempées pendant 20 minutes dans une solution (un poignet de cendre pour 101 d'eau) pour éliminer les charançons (Cylas formicarius) éventuellement présents sur les boutures. Un traitement insecticide a été réalisé avec le Décis entre le $30^{\text {ème }}$ et le $40^{\text {ème }}$ jour pour permettre de contrôler la mouche blanche et les autres ravageurs de la patate

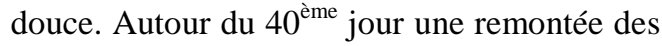
billons a été effectuée pour permettre aux racines tubéreuses, en croissance, de grossir dans de meilleures conditions à l'abri des attaques des charançons (Cylas formicarius). $\mathrm{Au}$ cours de la saison pluvieuse, l'irrigation fut faite en cas de besoin.

Le dispositif expérimental utilisé était un Split plot à quatre répétitions avec en parcelles principales les deux (02) variétés de patate douce (BF11, Ejumula 2) et en parcelles secondaires les six (06) traitements appliqués.

Les traitements suivants ont été retenus :

- T1: Aucun désherbage (témoin).

- T2: Sarclage manuel (SM) total et permanent.

- T3: Sarclage manuel de la quatrième $\left(4^{\text {ème }}\right)$ semaine suivant le repiquage jusqu'à la récolte.

- T4: Sarclage manuel (SM) de la sixième $\left(6^{\text {ème }}\right)$ semaine suivant le repiquage jusqu'à la récolte.

- T5: Sarclage manuel (SM) de la huitième $\left(8^{\text {ème }}\right)$ semaine suivant le repiquage jusqu'à la récolte.

- T6: Sarclage manuel (SM) de la dixième $\left(10^{\text {ème }}\right)$ semaine suivant le repiquage jusqu'à la récolte.

Ainsi, la variété locale de patate douce à chair blanche (BF 11) était nommée F0 et la variété de patate douce à chair orange (Ejumula 2) était nommée F1.

\section{Méthodologie \\ Inventaire de la macrofaune}

L'échantillonnage de la macrofaune a été effectué à partir de la huitième semaine après l'implantation de la culture par la méthode des monolithes, méthode standard TSBF. Le monolithe est un bloc du sol de 25 $\mathrm{cm} \times 25 \mathrm{~cm}$ x $30 \mathrm{~cm}$ de profondeur, prélevé dans chaque parcelle à l'aide d'un bloc métallique (Ayuke et al., 2009). Pour l'inventaire des termites, une fouille complémentaire à une profondeur de $5 \mathrm{~cm}$ est réalisée dans un transect de 5 x 2 autour du monolithe (Jones and Eggleton, 2000). Pour chaque traitement, trois (03) prélèvements de monolithes ont été effectués.

La macrofaune a été recueillie par tri à la main à l'aide de pincettes sur un plateau en plastique. Les espèces collectées, ont été conservées dans des flacons de conservation contenant de l'alcool à $75 \%$ et acheminés au laboratoire pour l'identification.

\section{Identification de la macrofaune et analyse des données}

L'identification de la macrofaune a été réalisée au Laboratoire d'Histoire Naturelle du Centre National de la Recherche Scientifique et Technologique au Burkina Faso (LHN/CNRST). Les macro-invertébrés ont été identifiés et dénombrés sous une loupe binoculaire à l'aide d'ouvrages de références et de clés dichotomiques.

\section{Analyse des données \\ Les données obtenues ont été} introduites dans le tableur Excel et soumises à l'analyse de variance (ANOVA), General Linear Model (GLM) en utilisant Minitab version 13.1. Les moyennes ont été comparées à l'aide du test de Fisher, au seuil de probabilité de 5\%. Pour la description de la macrofaune des sites, plusieurs paramètres ont été utilisés. La diversité spécifique a été exprimée par l'Indice de Shannon: 
$\mathrm{H}^{\prime}=-\Sigma\left(\right.$ pi $\ln$ pi), où $\mathrm{H}^{\prime}$ : indice de biodiversité de Shannon, $i$ : une espèce du milieu d'étude, $p_{i}$ : proportion d'une espèce $i$ par rapport au nombre total d'espèces $(\mathrm{S})$ dans le milieu d'étude (ou richesse spécifique du milieu), qui se calcule de la façon suivante: $p(i)=n_{i} / N$ où $n_{i}$ est le nombre d'individus pour l'espèce $i$ et $\mathrm{N}$ est l'effectif total (les individus de toutes les espèces). Cet indice est accompagné d'un autre indice, l'indice d'Equitabilité (IE), qui traduit l'abondance relative des différentes espèces au sein du peuplement : $\mathrm{IE}=\mathrm{H}^{\prime} / \mathrm{lnS}$, où $\mathrm{S}$ est la richesse spécifique du peuplement (Magurran, 2006). Un paramètre d'ordre quantitatif, l'abondance a été utilisée pour exprimer le nombre d'individus d'une espèce par unité de surface.

\section{RESULTATS}

\section{Macrofaune sous les traitements de la variété F0}

Au total, quinze (15) espèces d'insectes et deux (02) espèces de vers de terre ont été enregistrées sous les traitements de la variété F0 (Tableau 1). Pour les insectes, les espèces identifiées sont: Microtermes pusillus, Feronia sp, Capsus sp, Amara sp, Amara auliae, Philontus marginatus, Dromius sp, Dromius quadrimaculatus, Xestobium sp, Pachycondyla analis, Paederus sp, Trinervitermes sp, Monyomorium abyssinicumi, Dyschirius globusus, Amitermes stephensoni. Les vers de terre identifiés sont Milsonia inermis et Dichogaster affininis.

Le traitement $\mathrm{T} 1$ enregistre le plus grand nombre d'espèces (07) suivi du traitement T5 avec six (06) espèces. Les traitements T2 et T6 enregistre chacun 04 espèces; suivis de $\mathrm{T} 3$ avec 03 espèces et $\mathrm{T} 4$ avec 02 espèces. Les vers de terre ont enregistré dans tous les traitements à l'exception de T3. Le plus grand nombre d'individus par $\mathrm{m}^{2}$ est enregistré sous le traitement $\mathrm{T} 1$ avec vingt huit (28) individus constitués majoritairement de termites (Microtermes pusilus), suivis de T5 et T6 avec chacun onze (11) et quatorze (14) individus. T3 enregistre le plus petit nombre d'individus
(03) derrière T2 avec cinq (05) individus et T4 avec quatre (04) individus. L'analyse statistique a montré qu'il n'y a pas de différence significative entre les traitements $\mathrm{T} 2$, T3, et T4; ainsi que entre les traitements T5 et T6 (Figure 1). L'indice de diversité de Shannon (H') relève T2, T5 et T6 sont les traitements qui ont une macrofaune diversifiée avec des valeurs respectives de H' de 1,2621; 1,4447 et 1,2637. Le traitement $\mathrm{T} 4$ possède l'indice $H^{\prime}$ le plus petit avec 0,3046. Les traitements $\mathrm{T} 1$ et $\mathrm{T} 3$ se situent entre ces deux groupes avec des valeurs respectives de H' de 1,1429 et 1,0986. L'Indice d'Equitabilité (IE) montre que le traitement $\mathrm{T} 3$ est le lieu où la macrofaune a une répartition régulière avec IE = 1,0000 (Tableau 2). Les communautés les moins régulièrement reparties se trouvent sous $\mathrm{T} 1$ et T4 avec des valeurs respectives de IE de 0,3430 et 0,4056 . La macrofaune sous les traitements $\mathrm{T} 5$ et $\mathrm{T} 6$ ont une répartition moyenne avec des valeurs respectives de IE de 0,6065 et 0,52750 .

\section{Macrofaune sous les traitements de la variété F1}

Douze (12) espèces d'insectes et deux (02) espèces de vers de terre ont été enregistrées sous les traitements de la variété F1. Les espèces d'insectes identifiées sont: Microtermes pusillus, Feronia sp, Philontus marginatus, Pachycondyla senaarensis, Trinervitermes $\quad s p$, Tretramorium sericciventre, Spercheus emarginatus, Monyomorium bicolor, Macrotermes subhyalininis, Anthicus sp, Garucella sp, Ondontotermes. Milsonia inermis et Dichogaster affinis sont les deux (02) espèces de vers de terre identifiées.

Les vers de terre ont été rencontrés dans tous les traitements à l'exception du T1. Les deux espèces (Dichogaster affinis et Milsonia inermis) sont rencontrées en même temps uniquement au niveau de T5 (Tableau 3). Les traitements T5 enregistrent le plus grand nombre d'espèces de macrofaune (08) suivis de T1 et T6 avec chacun cinq (05) espèces. Le traitement $\mathrm{T} 2, \mathrm{~T} 3$ et $\mathrm{T} 4$ 
enregistrent chacun quatre (04) espèces. Le traitement T1 domine l'ensemble des traitements en nombre d'individus par unité de surface avec cinquante trois (53) individus. Ces individus sont majoritairement des termites (Microtermes pusillus). Suivent les traitements T6 et T5 avec respectivement 24 et 21 individus par $\mathrm{m}^{2}$. Les traitements $\mathrm{T} 3$ et T4 enregistrent chacun dix (10) individus par unité de surface. T2 est le traitement qui enregistre le plus faible nombre d'individus par unité de surface avec huit (08) individus. L'analyse statistique ne montre pas de différence significative entre les traitements T2, T3 et T4 (Figure 2). Par contre, nous avons une différence significative entre ces 03 traitements avec le traitement $\mathrm{T} 1$ d'une part et les traitements T5 et T6 d'autre part. Les valeurs de l'indice de diversité place le traitement T5 en tête des autres traitements avec $\mathrm{H}^{\prime}=1,8590$ (Tableau 4). Ce traitement est en même temps le traitement où les communautés de macrofaunes sont régulièrement reparties avec $\mathrm{IE}=0,6106$. Les traitements $\mathrm{T} 1$ et $\mathrm{T} 2$ ont des valeurs de $\mathrm{H}^{\prime}$ respectives de 1,2488 et 1,2352. Les traitements $\mathrm{T} 3$ et $\mathrm{T} 4$ sont également des traitements de même niveau de diversité avec respectivement 1,1935 et 1,1596 . Le traitement le moins diversifiée est T6 avec $\mathrm{H}^{\prime}=1,0042$.

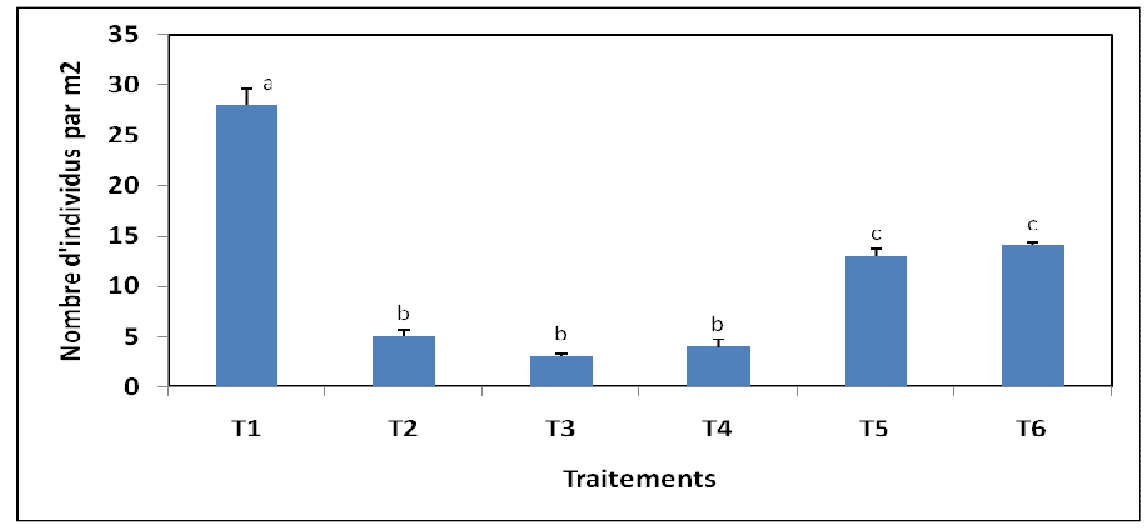

Figure 1 : Nombre d'individus par unité de surface des traitements de la variété F0. Les histogrammes portant une même lettre ne sont pas significativement différents au seuil $\mathrm{p}<0,05$, selon le test de Fisher.

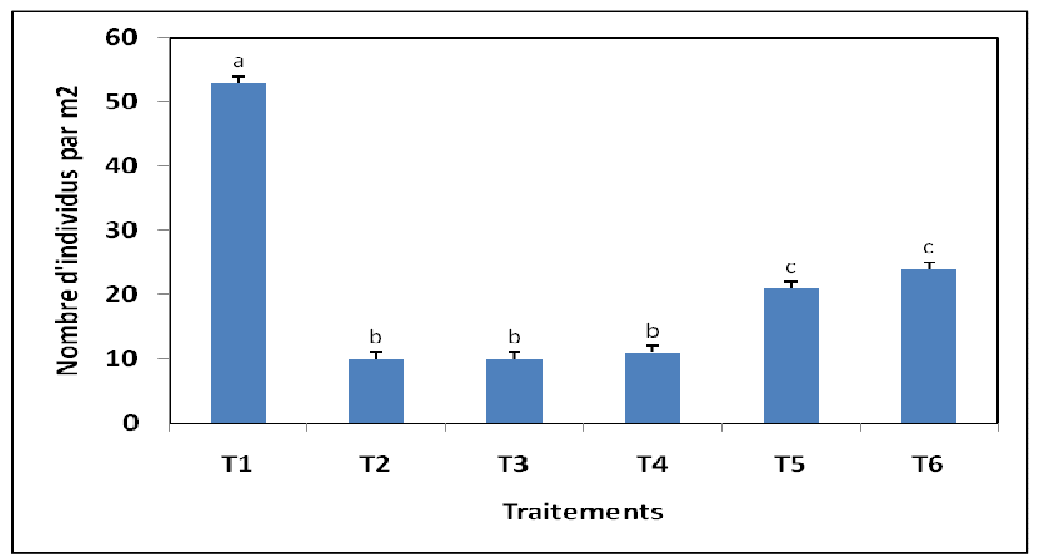

Figure 2 : Nombre d'individus par unité de surface des traitements de la variété F1. Les histogrammes portant une même lettre ne sont pas significativement différents au seuil $\mathrm{p}<0,05$, selon le test de Fisher. 
Tableau 1 : Macrofaune sous différents types de traitements de la variété F0.

\begin{tabular}{|c|c|c|c|c|c|}
\hline Traitements & Ordre & Famille & Genre & Espèce & $\begin{array}{c}\text { Nombre } \\
\text { par unité de } \\
\text { surface }\end{array}$ \\
\hline & Hyménoptères & Termitidae & Microtermes & pusillus & $20 \pm 1,2$ \\
\hline \multirow[t]{10}{*}{$\mathbf{T 1}$} & Coléoptères & Carabidae & Feronia & $\mathrm{sp}$ & $1 \pm 0,06$ \\
\hline & Hémiptères & Miridae & Capsus & $\mathrm{sp}$ & $1 \pm 0,06$ \\
\hline & Coléoptères & Carabidae & Amara & auliae & $1 \pm 0,06$ \\
\hline & Coléoptères & Staphylinidae & Philonthus & marginatus & $2 \pm 0,12$ \\
\hline & Vers de terre & Acanthodrilidae & Milsonia & inermis & $1 \pm 0,06$ \\
\hline & Coléoptères & Carabidae & Dromius & $\mathrm{sp}$ & $1 \pm 0,06$ \\
\hline & Coléoptères & Anobiidae & Xestobium & $\mathrm{sp}$ & $1 \pm 0,06$ \\
\hline & Vers de terre & Octochaeitidae & Dichogaster & affinis & $2 \pm 0,12$ \\
\hline & Hyménoptères & Formicidae & Pachycondyla & analis & $1 \pm 0,06$ \\
\hline & Coléoptères & Carabidae & Dromius & $\mathrm{sp}$ & $1 \pm 0,06$ \\
\hline $\mathrm{T} 2$ & Vers de terre & Acanthodrilidae & Milsonia & inermis & $1 \pm 0,06$ \\
\hline \multirow[t]{3}{*}{ T3 } & Coléoptères & Carabidae & Dromius & $\mathrm{sp}$ & $1 \pm 0,06$ \\
\hline & Coléoptères & Staphylinidae & Paederus & $\mathrm{sp}$ & $1 \pm 0,06$ \\
\hline & Coléoptères & Staphylinidae & Philonthus & marginatus & $1 \pm 0,06$ \\
\hline \multirow[t]{4}{*}{ T4 } & Hyménoptères & Termitidae & Trinervitermes & $\mathrm{sp}$ & $3 \pm 0,27$ \\
\hline & Vers de terre & Acanthodrilidae & Milsonia & inermis & $1 \pm 0,06$ \\
\hline & Hyménoptères & Formicidae & Monomorium & abyssinicumi & $1 \pm 0,06$ \\
\hline & Coléoptères & Carabidae & Dromius & $\mathrm{sp}$ & $1 \pm 0,06$ \\
\hline \multirow[t]{5}{*}{ T5 } & Coléoptères & Carabidae & Dyschirius & globusus & $1 \pm 0,06$ \\
\hline & Hyménoptères & Formicidae & Amitermes & stephensoni & $2 \pm 0,12$ \\
\hline & Coléoptères & Carabidae & Amara & auliae & $5 \pm 0,36$ \\
\hline & Vers de terre & Acanthodrilidae & Milsonia & inermis & $1 \pm 0,06$ \\
\hline & Coléoptères & Carabidae & Amara & $\mathrm{sp}$ & $4 \pm 0,31$ \\
\hline \multirow[t]{3}{*}{ T6 } & Hyménoptères & Termitidae & Trinervitermes & $\mathrm{sp}$ & $7 \pm 0,44$ \\
\hline & Vers de terre & Acanthodrilidae & Milsonia & inermis & $2 \pm 0,12$ \\
\hline & Vers de terre & Octochaeitidae & Dichogaster & affinis & $1 \pm 0,06$ \\
\hline
\end{tabular}

Tableau 2 : Indice de Shannon et de l'Indice d'Equitabilité par traitement (variété F0).

\begin{tabular}{lcccccc}
\hline Traitements & T1F0 & T2F0 & T3F01 & T4F0 & T5F0 & T6F0 \\
\hline Indice de Shannon (H') & 1,1429 & 1,2621 & 1,0986 & 0,5623 & 1,4447 & 1,0042 \\
Indice d'Equitabilité (IE) & 0,3430 & 0,7842 & 1,000 & 0,4056 & 0,6065 & 0,5275 \\
\hline
\end{tabular}


Tableau 3 : Macrofaune sous différents types de traitements de la variété F1.

\begin{tabular}{lccccc}
\hline Traitements & Ordre & Famille & Genre & Espèce & $\begin{array}{c}\text { Nombre par } \\
\text { unité de } \\
\text { surface }\end{array}$ \\
\hline T1 & Hyménoptères & Formicidae & Pachycondyla & senaarensis & $3 \pm 0,16$ \\
& Hyménoptères & Termitidae & Microtermes & pusillus & $29 \pm 1,74$ \\
& Coléoptères & Staphylinidae & Philontus & marginatus & $3 \pm 0,17$ \\
& Hyménoptères & Formicidae & Tetramorium & sericciventre & $11 \pm 0,64$ \\
& Hyménoptères & Termitidae & Trinivertemes & sp & $7 \pm 0,40$ \\
\hline T2 & Vers de terre & Octochaeitidae & Dichogaster & affinis & $2 \pm 0,12$ \\
& Coléoptères & Hydrophilidae & Spercheus & marginatus & $2 \pm 0,12$ \\
& Hyménoptères & Formicidae & Monomorium & bicolor & $3 \pm 0,17$ \\
& Hyménoptères & Formicidae & Pachycondyla & senaarensis & $1 \pm 0,06$ \\
\hline \multirow{3}{*}{ T3 } & Coléoptères & Carabridae & Feronia & sp & $1 \pm 0,06$ \\
& Vers de terre & Octochaeidae & Dichogaster & affinis & $4 \pm 0,23$ \\
& Hyménoptères & Formiadae & Monomorium & bicolor & $1 \pm 0,06$ \\
& Hyménoptères & Termitidae & Macrotermes & subhyalinis & $4 \pm 0,23$ \\
\hline T4 & Hyménoptères & Formicidae & Tetramorium & sericciventre & $1 \pm 0,06$ \\
& Vers de terre & Octochaeitidae & Dichogaster & affinis & $7 \pm 0,40$ \\
& Hyménoptères & Termitidae & Macrotermes & subhyalinis & $1 \pm 0,06$ \\
& Coléoptères & Scarabaeidae & larve & & $1 \pm 0,06$ \\
& Hyménoptères & Formiadae & Pachycondyla & senaarensis & $1 \pm 0,06$ \\
\hline & & & & &
\end{tabular}

Tableau 4 : Indice de Shannon et Indice d'Equitabilité par traitement (variété F1).

\begin{tabular}{lllllll}
\hline Traitement & T1F1 & T2F1 & T3F1 & T4F1 & T5F1 & T6F1 \\
\hline Indice de Shannon (H') & 1,2488 & 1,2352 & 1,1935 & 1,1596 & 1,8590 & 1,4447 \\
Indice d'Equitabilité (IE) & 0,3145 & 0,5940 & 0,5184 & 0,4836 & 0,6106 & 0,4546 \\
\hline
\end{tabular}

\section{DISCUSSION}

Le sol est un milieu vivant constitué de milliers d'espèces représentées. La macrofaune se compose d'un large panel d'organismes dont des auxiliaires de cultures.
Elle réside dans le sol pour la majeure partie de sa vie et est donc extrêmement sensible au type de travail du sol. Le type de technique choisi pour travailler le sol va avoir des impacts importants sur les organismes et les 
interactions entre toutes ses composantes (Rossi, 2006 ; Crittenden et al., 2014). Sous culture des deux variétés de patate douce, notre étude a inventorié quinze (15) espèces d'insectes et deux (02) espèces de vers de terre. Nos résultats montrent que la composition des populations de la faune du sol diffère selon le type de traitement appliqué. Le groupe des insectes est dominé par les termites représentés par des termites moissonneurs (Trinervitermes. sp.), et des lignivores ou xylophages (M. pusillus). Nos résultats montrent que les termites semblent donc présenter une meilleure adaptation dans ce milieu mis en culture. Les termites moissonneurs qui se nourrissent des feuilles de litière et des tiges de graminées et les termites lignivores qui se nourrissent de bois morts en décomposition ont trouvé une source de nourriture supplémentaire dans le sol non désherbé. Ces apports ont contribué à augmenter le nombre d'individus par unité de surface. Ces résultats corroborent ceux de nombreux auteurs qui avait montré que les termites sont dominants sur des terres longtemps misent en culture en milieu tropical (Maldague, 2003 ; Traoré, 2012). Maldague (2003) en particulier a montré que les termites sont dominants sur des terres longtemps misent en culture et contribuent à près de 74 $\%$ de la densité totale des termites. De manière générale, on a montré que dans les écosystèmes tropicaux et sub- tropicaux, la macrofaune est essentiellement représentée par les termites, les fourmis et les vers de terre, avec des abondances respectives de $37 \%, 23 \%$ et $9 \%$ (Lavelle et al., 2006).

Nos résultats montrent aussi que le type de technique choisi pour travailler le sol a eu d'importants effets sur la densité des organismes du sol. Les traitements sans sarclage et ceux dont le sarclage a lieu à partir de la huitième semaine sont ceux qui ont eu le plus grande nombre d'individus quelle que soit la variété de patate douce. Pour la variété F0, on passe de 28 individus par unité de surface pour T1 à en moyenne 3 individus par unité de surface pour T2, T3 et T4; soit une baisse de $82 \%$. Par rapport aux T4 et T5, cette baisse est de $61 \%$; on passe de 28 individus par unité de surface à en moyenne 13 individus par unité de surface. Au niveau de la variété $\mathrm{F} 1$, on passe de 53 individus par unité de surface pour $\mathrm{T} 1$ à en moyenne 10 individus par unité de surface pour les traitements T2, $\mathrm{T} 3$ et T4 soit une baisse de $82 \%$. Cette baisse est $41,51 \%$ par rapport à T5 et T6. Nos résultats corroborent ceux de nombreux auteurs qui ont montré que la mise en culture des sols peut se traduire par une diminution de l'abondance et la diversité de la faune des sols (Mathieu, 2004 ; Rousseau et al., 2014) ou une modification de sa composition (Ruiz Camacho, 2004 ; Ponges et al., 2013 ; Pelosi et al., 2014). Le traitement $\mathrm{T} 5$ est le lieu où la faune du sol est la plus diversifiée pour les 02 variétés avec respectivement $\mathrm{H}^{\prime}=1,4447$ et $H^{\prime}=1,8590$. Les traitements $\mathrm{T} 1$ pour les deux variétés sont les traitements où l'Indice d'Equitabilité est le plusfaible $(0,3430$ et 0,3145), caractéristique d'une communauté dominée majoritairement par une seule espèce. Pour les deux variétés, on observe une différence significative entre trois groupes de traitements : T1, (T2, T3, T4) et T5, T6. On n'observe pas de différence significative entre le type des traitements par rapport au type de variété. Pour les six traitements de la variété F0, nous avons des valeurs respectives de $\mathrm{p}$ value de 0,$731 ; 0,892 ; 0,376 ; 0,273 ; 0,423$ et 0,233 . Pour la variété $F 1$, ces valeurs sont de 0,$362 ; 0,820 ; 0,353 ; 0,134 ; 0,082 ; 0,503$ et 0,455 . Cette analyse statistique montre donc que nos résultats traduisent essentiellement un effet des traitements; on n'observe pas un effet du type de variété de patate. Nos 
résultats corroborent ceux des travaux antérieurs qui ont montré que le type de technique choisi pour travailler le sol a des impacts importants sur les organismes du sol (Lavelle et Fragoso, 2000 ; Peres et al., 2008 ; Traoré, 2012 ; Ponge et al., 2013). Certaines communautés de macrofaunes ont été plus favorisées par rapport à d'autres.

\section{Conclusion}

Nous avons étudié au cours de ce travail les effets sur la macrofaune du sol de six traitements appliqués sous la culture de deux variétés de patate douce. Les six traitements pratiqués ont montré des impacts différents sur le maintien des communautés de la macrofaune du sol. Pour les deux variétés, l'absence totale de désherbage a favorisé beaucoup plus l'installation des termites qui semblent bénéficier beaucoup plus de ce type de traitement. Les autres traitements ont connu l'installation d'une faune de sol de composition plus ou moins équilibrée. Il n'a pas été observé une influence du type de variété de patate douce sur la macrofaune du sol.

\section{REMERCIEMENTS}

Nous remercions les collègues birba SIBIRI, Ali SAWADOGO, Mme NIKIEMA qui ont activement participé à la collecte des données sur le terrain.

\section{CONFLITS D'INTERETS}

Les auteurs déclarent qu'il n y a aucun conflit d'intérêt.

\section{REFERENCES}

Ayuke FO, Karanja NK, Muya EM, Musombi BK, Mungatu J, Nyamasyo. 2009. Macro-fauna diversity and abundance across different land use systems in
Embu, Kenya. Trop. Subtrop. Agroecosyst., 11: 371-384.

Barros E, Grimaldi M, Sarrazin M, Chauvel A, Mitja D, Desjardins T, Lavelle P. 2004. Soil physical degradation and changes in macrofaunal communities in Central Amazon. Applied Soil Ecology, 26: 157-168. DOI:10.1016/j.apsoil. 2003.10.012

Blouina M, Hodson M E, Delgado E A, Baker G, Brussaard L, Butt KR, Dai J, Dendooven L, Peres G, Tondoh J E, Cluzeau D, Brun JJ. 2013. A review of earthworm impact on soil function and ecosystem services. European Journal of Soil Science, 64: 161-182. DOI: 10.1111/ejss. 12025

Boyer J, Reversat G, Lavelle P, Chabanne A. 2013. Interactions between earthworms and plant-parasitic nematodes. European Journal of Soil Biology, 59: 43-47. DOI: http://dx.doi.org/ 10.1016/j.ejsobi.2013.10.004

Chaudhuri PS, Pal TK, Nath S, Dey SK. 2012. Effects of five earthworm species on some physico-chemical properties of soil. J. Environ. Biol., 33: 713-716.

Crittenden SJ, Eswaramurthy T, De Goede R GM, Brussaard L, Pulleman MM. 2014. Effect of tillage on earthworms over short- and medium-term inconventional and organic farming. Applied Soil Ecology, 83: 140-148. DOI: http://dx.doi.org/10.1016/ j.apsoil.2014.03.001

Decaëns T, Jiménez JJ, Gioia CGJ, Measey GJ, Lavelle P. 2006. The values of soil animals for conservation biology. European Journal of Soil Biology, 42: S23-S38.

DOI::10.1016/j.ejsobi.2006.07.001

Huhta V. The role of soil fauna in ecosystems: A historical review. Pedobiologia, 50: 
489-495. DOI:10.1016/j.pedobi.2006. 08.006

Jones DT, Eggleton P. 2000. Sampling termite assemblages in tropical forests : testing a rapid biodiversity assessment protocol. Journal of Applied Ecology, 37: 191-203.

Jouquet PP, Podwojewski P, Bottinelli N, Mathieu J, Orange D, Tran DT, Valentin C. 2007. Impact du changement d'usage des sols sur la biodiversité : Conséquences sur l'érosion des sols. Actes des JSIRAUF, Hanoi, 6-9 novembre 2007, 1-6.

Jouquet P, Traoré S, Choosai C, Hartmann C, Bignell D. 2011. Influence of termites on ecosystem functioning. Ecosystem services provided by termites. European Journal of Soil Biology, 47: 215-222. DOI:10.1016/j.ejsobi.2011. 05.005

Jouquet P, Blanchart E, Capowiez Y. 2014. Utilization of earthworms and termites for the restoration of ecosystem functioning. Applied Soil Ecology, 73: 34- 40. DOI:http://dx.doi.org/10.1016/ j.apsoil.2013.08.004

Lafont A, Risède J-M, Loranger-Merciris G, Clermont-Dauphin C, Dorel M, Rhino B, Lavelle P. 2007. Effects of the earthworm Pontoscolex corethrurus on banana plants infected or not with the plant-parasitic nematode Radopholus similis. Pedobiologia, 51: 311-318. DOI:10.1016/j.pedobi.2007.05.004

Lavelle P, Fragoso C. 2000. La macrofaune $\mathrm{du}$ sol: une ressource en danger dans un monde en changement. IBOY 2000 (International Biodiversity Observation Year), Rapport de colloque, Bondy, France, $44 \mathrm{p}$.

Lavelle P, Decaens M, Aubert M, Barot S, Bloiun M, Bureau F, Magerie P, Mora
P, Rossi J-P. 2006. Soil invertebrates and ecosystem services. European Journal of Soil Biology, 42(1): S3-S15.

Maldague M. 2003. Études des termites de la région de Bambesa (Uele, RDC) en relation avec la matière organique du sol. Edition Bibliothèque Paul-ÉmileBoulet de l'Université du Québec à Chicoutimi.

Pelosi C, Peya B, Hedde M, Caroa G, Capowiez Y, Guernion M, Peigné J, Piron D, Bertrand M, Cluzeau D. 2014. Reducing tillage in cultivated fields increases earthworm functionaldiversity. Applied Soil Ecology, 83: 79-87. DOI: http://dx.doi.org/10.1016/j.apsoil.2013. 10.005

Peres G, Piron D, Bellido A, Goater C, Cluzeau D. 2008. Earthworms used as indicators of agricultural managements. Fresenius Environmental Bulletin, 17(8b): 1181-1189.

Ponge J-F, Pérès G, Guernion M, RuizCamacho N, Cortet J, Pernin C, Villenave C, Chaussod R, MartinLaurent F, Bispo A, Cluzeau D. 2013. The impact of agricultural practices on soil biota: A regional study. Soil Biology \& Biochemistry, 67: 271-284. DOI: $\quad$ http://dx.doi.org/10.1016/j. soilbio.2013.08.026

Rombke J, Sousa JP, Schouten T, Rieper TF. 2006. Monitoring of soil organisms: a set of standardized field methods proposed by ISO. European Journal of Soil Biology, 42(1): S61-S63.

Rossi J-P. 2006. Soil invertebrates and ecosystem services. European Journal of Soil Biology, 42(1): S3-S15.

Rousseau L, Fonte SJ, Téllez O, Van Der Hoek R, Lavelle P. 2013. Soil macrofauna as indicators of soil quality 
and land use impacts in smallholder agroecosystems of western Nicaragua. Ecological Indicators, 27: 71-82. DOI: http://dx.doi.org/10.1016/ j.ecolind.2012.11.020

Ruiz Camacho N. 2004. Mise au point d'un système de bioindication de la qualité du sol basé sur l'étude des peuplements de macroinvertébrés. Thèse de Doctorat, Université de Paris 6, France, $327 \mathrm{p}$.

Traoré M. 2012. Impact des pratiques agricoles (rotation, fertilisation et labour) sur la dynamique de la microfaune et la macrofaune du sol sous culture de sorgho et de niébé au
Centre Ouest du Burkina Faso. Thèse de Doctorat Unique, Science du Sol, Institut du Développement Rural, Université Polytechnique de BoboDioulasso, Burkina Faso. 169p.

Wolters V. 2001. Biodiversity of soil animals and its function. Eur. J. Soil Biol., 37: 221-227. DOI: S1164556301010883/ FLA

Zangerlé A. 2011. Participation des organismes ingénieurs à l'agrégation des sols: analyse des patrons et mise en évidence des interactions. Thèse de Doctorat, Université Pierre et Marie Curie. 170p. 